\title{
Strange Duality Between the Quadrangle Complete Intersection Singularities
}

\author{
Wolfgang Ebeling $^{1}$ (D) Atsushi Takahashi ${ }^{2}$
}

Received: 26 February 2021 / Revised: 31 May 2021 / Accepted: 2 June 2021 / Published online: 22 June 2021

(C) The Author(s) 2021

\begin{abstract}
There is a strange duality between the quadrangle isolated complete intersection singularities discovered by the first author and Wall. We derive this duality from a variation of the Berglund-Hübsch transposition of invertible polynomials introduced in our previous work about the strange duality between hypersurface and complete intersection singularities using matrix factorizations of size two.
\end{abstract}

Keyword Mirror symmetry, Singularity, Reduction, Strange duality, Dolgachev numbers, Gabrielov numbers

Mathematics Subject Classification 32S20 · 32S30 · 14J33

\section{Introduction}

Arnold [2] observed a strange duality between the 14 exceptional unimodal singularities. It is well known that this duality is a special case of the Berglund-Hübsch duality of invertible polynomials, see e.g. [12]. Wall and the first author [15] discovered an extension of this duality embracing on one hand series of bimodal hypersurface singularities and on the other hand, certain isolated complete intersection singularities (ICIS) in $\mathbb{C}^{4}$. The duals of these ICIS are not themselves singularities but are virtual $(k=-1)$ cases of series (e.g. $J_{3, k}, k \geq 0$ ) of bimodal singularities. In [15], the $k=-1$ cases of the series were called virtual singularities and Milnor lattices were

Wolfgang Ebeling

ebeling@math.uni-hannover.de

Atsushi Takahashi

takahashi@math.sci.osaka-u.ac.jp

1 Institut für Algebraische Geometrie, Leibniz Universität Hannover, Postfach 6009, 30060

Hannover, Germany

2 Department of Mathematics, Graduate School of Science, Osaka University, Toyonaka, Osaka 560-0043, Japan 
Table 1 The elements with $k=0$ of the series

\begin{tabular}{lllll}
\hline Name & Equations & Restrictions & Dol & Weights \\
\hline$J_{2,0}^{\prime}$ & $\left(x y+w^{2}, a x^{5}+x w^{2}+y w+z^{2}\right)$ & $a \neq 0,-\frac{4}{27}$ & $2,2,2,6$ & $2,6,5,4 ; 8,10$ \\
$K_{1,0}^{\prime}$ & $\left(x y+w^{2}, a x^{4}+x w^{2}+y^{2}+z^{2}\right)$ & $a \neq 0, \frac{1}{4}$ & $2,2,4,4$ & $2,4,4,3 ; 6,8$ \\
$L_{1,0}$ & $\left(x y+z w, a x^{4}+x w^{2}+y w+z^{2}\right)$ & $a \neq 0,-1$ & $2,2,3,5$ & $2,5,4,3 ; 7,8$ \\
$M_{1,0}$ & $\left(2 x y+w^{2}-z^{2}, a x^{2} z+x^{2} w+2 y w\right)$ & $a \neq 0, \pm 1$ & $2,3,3,4$ & $2,4,3,3 ; 6,7$ \\
$I_{1,0}$ & $\left(x(y-z)+w^{3}, a w^{3}+y(z-x)\right)$ & $a \neq 0,1$ & $3,3,3,3$ & $3,3,3,2 ; 6,6$ \\
\hline
\end{tabular}

associated to them, but they do not coincide with the Milnor lattices of the germs at the origin by setting $k=-1$ in Arnold's equations of the series, which are exceptional unimodal singularities with a smaller Milnor number. In [13], we showed that the virtual singularities exist in the sense that the equations have to be considered as global polynomials and we derived this extension from the mirror symmetry and the Berglund-Hübsch duality of invertible polynomials.

Arnold's 14 exceptional unimodal singularities are triangle hypersurface singularities, i.e., they are weighted homogeneous singularities obtained from triangles in the hyperbolic plane. More precisely, they are determined by triangles with angles $\frac{\pi}{b_{1}}, \frac{\pi}{b_{2}}, \frac{\pi}{b_{3}}$, where $b_{1}, b_{2}, b_{3}$ are positive integers called the Dolgachev numbers of the singularity. The $k=0$ elements of the bimodal series are quadrangle hypersurface singularities, i.e., they are related in a similar way to quadrangles in the hyperbolic plane. They are determined by four positive integers $b_{1}, b_{2}, b_{3}, b_{4}$. For 6 quadruples $\left(b_{1}, b_{2}, b_{3}, b_{4}\right)$, the corresponding quadrangle singularities are hypersurface singularities. They are the $k=0$ elements of the 8 series of bimodal hypersurface singularities. The dual ICIS are triangle complete intersection singularities in $\mathbb{C}^{4}$. There are 8 of them determined by 8 triples $\left(b_{1}, b_{2}, b_{3}\right)$. For another 5 quadruples $\left(b_{1}, b_{2}, b_{3}, b_{4}\right)$, the quadrangle singularities are ICIS. They are again the $k=0$ elements of certain series of singularities. These series are the 8 series of $\mathcal{K}$-unimodal ICIS in Wall's classification [21]. Wall and the first author also observed a duality between the $k=-1$ cases of these series (see also [6, Sect. 3.6]). They were called virtual singularities as well. The objective of this paper is to show that these singularities exist as well and to derive this duality from the Berglund-Hübsch duality, too. The equations and notations of these quadrangle complete intersection singularities according to [21] together with their Dolgachev numbers and systems of weights are listed in Table 1. The virtual singularities obtained by setting $k=-1$ in the equations of Wall [21] are listed in Table 2.

We derive this duality from our paper [13]. An important tool are matrix factorizations of size two. In [14], we showed that such a matrix factorization can be considered as an inverse to Wall's reduction of complete intersection singularities to hypersurface singularities [22]. We proceed as follows. In [13], we classified certain $4 \times 3$-matrices which provided the duality to complete intersection singularities. Here we consider the polynomials determined by these matrices for the bimodal series. We determine the matrix factorizations of size two of the corank 3 polynomials. It turns out that we get exactly 8 possibilities which correspond to the 8 series of ICIS. We show that one can associate $4 \times 4$-matrices to these equations such that the duality is given by 
Table 2 Setting $k=-1$ in Wall's equations

\begin{tabular}{ll}
\hline Name & Equation \\
\hline$J_{2,-1}^{\prime}$ & $\left(x y+w^{2}, x^{4}+x w^{2}+y w+z^{2}\right)$ \\
$K_{1,-1}^{\prime}$ & $\left(x y+w^{2}, x^{3}+x w^{2}+y^{2}+z^{2}\right)$ \\
$K_{1,-1}^{b}$ & $\left(x y+w^{2}, x^{4}+x^{2} w+2 x w^{2}+y^{2}+z^{2}\right)$ \\
$L_{1,-1}$ & $\left(x y+z w, x^{3}+x w^{2}+y w+z^{2}\right)$ \\
$L_{1,-1}^{\sharp}$ & $\left(x y+z w,-x^{4}+x^{2} w+x w^{2}+y w+z^{2}\right)$ \\
$M_{1,-1}$ & $\left(2 x y+w^{2}-z^{2}, z^{2}+x^{2} w+2 y w\right)$ \\
$M_{1,-1}^{\sharp}$ & $\left(2 x y+w^{2}-z^{2}, z^{2}+x^{2}(w-z)+2 y w\right)$ \\
$I_{1,-1}$ & $\left(x(y-z)+w^{3}, x w+y(z-x)\right)$ \\
\hline
\end{tabular}

the Berglund-Hübsch transposition of these matrices. Using the definition of the virtual singularities in [13] and matrix factorizations again, we define virtual quadrangle complete intersection singularities.

Similarly as in [13], we associate Dolgachev numbers to the virtual singularities. These are two pairs of numbers corresponding to a decomposition of the equations into two parts. We also associate Gabrielov numbers to the virtual singularities by considering deformations to cusp singularities. We consider the second function on the zero set of the first function. Again this function has to be considered as a global function. It turns out that these functions have, besides an isolated critical point at the origin, additional critical points outside the origin. We consider Coxeter-Dynkin diagrams of distinguished bases of thimbles corresponding to these functions taking the additional critical points into account.

We show that the Dolgachev numbers of a virtual singularity are the Gabrielov numbers of the dual one, and vice versa. Moreover, we show that an analogue of [13, Theorem 6] holds: the product $\prod_{j=1}^{2} \bar{\zeta}_{X, j}(t)$ of the reduced zeta functions of the monodromies of a virtual singularity $X$ coincides with the product of the Poincare series $P_{\widetilde{X}_{0}}(t)$ and a polynomial $\mathrm{Or}_{\tilde{X}_{0}}(t)$ related to the Dolgachev numbers of the $k=0$ element $\widetilde{X}_{0}$ of the dual series. The results are summarized in Table 3. Here we use for a rational function $\prod_{m \mid h}\left(1-t^{m}\right)^{\chi_{m}}\left(\chi_{m} \in \mathbb{Z}\right)$ the symbolic notation $\prod_{m \mid h} m^{\chi_{m}}$. For more details we refer to Sect. 7 .

\section{Invertible Polynomials}

We recall some general definitions.

A complete intersection singularity in $\mathbb{C}^{n}$ given by polynomial equations $f_{1}=$ $\cdots=f_{k}=0$ is called weighted homogeneous if there are positive integers $w_{1}, \ldots, w_{n}$ (called weights) and $d_{1}, \ldots, d_{k}$ (called degrees) such that $f_{j}\left(\lambda^{w_{1}} x_{1}, \ldots, \lambda^{w_{n}} x_{n}\right)=$ $\lambda^{d_{j}} f_{j}\left(x_{1}, \ldots, x_{n}\right)$ for $j=1, \ldots, k$ and for $\lambda \in \mathbb{C}^{*}$. We call $\left(w_{1}, \ldots, w_{n} ; d_{1}, \ldots, d_{k}\right)$ a system of weights.

A weighted homogeneous polynomial $f\left(x_{1}, \ldots, x_{n}\right)$ is called invertible if it can be written 


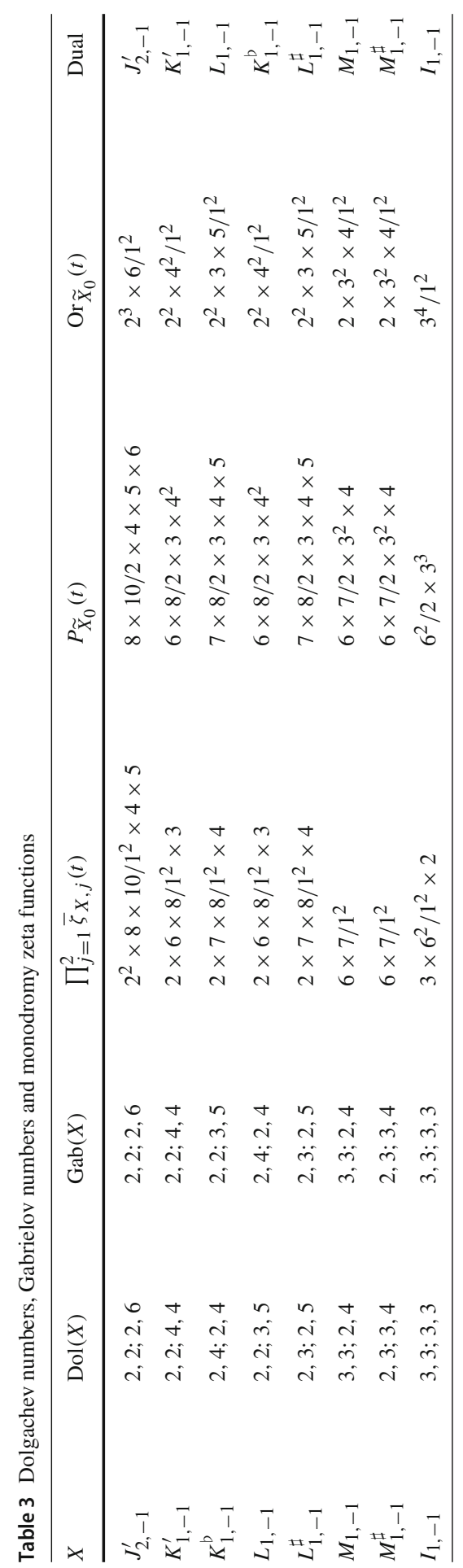




$$
f\left(x_{1}, \ldots, x_{n}\right)=\sum_{i=1}^{n} a_{i} \prod_{j=1}^{n} x_{j}^{E_{i j}},
$$

where $a_{i} \in \mathbb{C}^{*}, E_{i j}$ are non-negative integers, and the $n \times n$-matrix $E:=\left(E_{i j}\right)$ is invertible over $\mathbb{Q}$.

An invertible polynomial is called non-degenerate if it has an isolated singularity at the origin.

Remark 1 Note that sometimes an invertible polynomial is defined to be a nondegenerate invertible polynomial, see e.g. [12].

Let $f$ be an invertible polynomial given as above. By rescaling of the variables, one can assume that $a_{i}=1$ for $i=1, \ldots, n$. Moreover, we can assume that $\operatorname{det} E>0$.

The Berglund-Hübsch transpose [4] $\widetilde{f}$ of $f$ is defined by the transpose matrix $E^{\mathrm{T}}$ of $E$, i.e.

$$
\tilde{f}\left(x_{1}, \ldots, x_{n}\right)=\sum_{i=1}^{n} a_{i} \prod_{j=1}^{n} x_{j}^{E_{j i}} .
$$

Let $f\left(x_{1}, \ldots, x_{n}\right)$ be an invertible polynomial. The canonical system of weights $W_{f}$ of $f$ is the system of weights $\left(w_{1}, \ldots, w_{n} ; d\right)$ given by the unique solution of the equation

$$
E\left(\begin{array}{c}
w_{1} \\
\vdots \\
w_{n}
\end{array}\right)=\operatorname{det}(E)\left(\begin{array}{c}
1 \\
\vdots \\
1
\end{array}\right), \quad d:=\operatorname{det}(E)
$$

We define

$$
q_{1}:=\frac{w_{1}}{d}, \ldots, q_{n}:=\frac{w_{n}}{d}
$$

The maximal group of diagonal symmetries of $f$ is the group

$$
G_{f}=\left\{\left(\lambda_{1}, \ldots, \lambda_{n}\right) \in\left(\mathbb{C}^{*}\right)^{n} \mid f\left(\lambda_{1} x_{1}, \ldots, \lambda_{n} x_{n}\right)=f\left(x_{1}, \ldots, x_{n}\right)\right\} .
$$

It always contains the exponential grading operator

$$
g_{0}:=\left(e^{2 \pi i q_{1}}, \ldots, e^{2 \pi i q_{n}}\right) .
$$

Denote by $G_{0}$ the subgroup of $G_{f}$ generated by $g_{0}$.

By [3] (see also [10, Proposition 2]), $\operatorname{Hom}\left(G_{f}, \mathbb{C}^{*}\right)$ is isomorphic to $G_{\tilde{f}}$. For a subgroup $G \subset G_{f}$, Berglund and Henningson [3] defined its dual group $\widetilde{G}$ by

$$
\widetilde{G}:=\operatorname{Hom}\left(G_{f} / G, \mathbb{C}^{*}\right) .
$$




\section{Wall's Reduction}

Let $(X, 0)$ be an ICIS in $\mathbb{C}^{4}$ given by an equation $F=0$ where

$$
F(x, y, z, w)=(x y-a(z, w), y b(z, w)+c(x, z, w))
$$

where $a(z, w)$ and $c(x, z, w)$ are polynomials of degree $\geq 2, b(z, w)$ is a polynomial of degree $\geq 1$, and $x, b(z, w)$ form a regular sequence in $\mathbb{C}[x, z, w]$. Then we can consider the reduction

$$
L_{y} F(x, z, w)=x c(x, z, w)+a(z, w) b(z, w)
$$

of [22] corresponding to the variable $y$. This means that we eliminate the variable $y$ to get the equation of a hypersurface singularity in $\mathbb{C}^{3}$. Geometrically, this elimination corresponds to the projection along the $y$-axis on the coordinate space of the remaining variables $x, z, w$. It is proved in [22, Theorem 7.9], for the case $b(z, w)=z$, that the Milnor number increases by one.

In [14], we considered certain polynomials of the form

$$
f(x, z, w)=x c(x, z, w)+a(z, w) b(z, w)
$$

with the conditions on $a(z, w), b(z, w)$, and $c(x, z, w)$ as above and associated a complete intersection singularity to a graded matrix factorization of size two of $f$. We showed that, in this way, we get an inverse to Wall's reduction. More precisely, a matrix factorization (of size two) of $f$ is given by two matrices

$$
q_{0}=\left(\begin{array}{cc}
a(z, w) & -x \\
c(x, z, w) & b(z, w)
\end{array}\right) \text { and } q_{1}=\left(\begin{array}{cc}
b(z, w) & x \\
-c(x, z, w) & a(z, w)
\end{array}\right)
$$

such that

$$
q_{0} q_{1}=q_{1} q_{0}=\left(\begin{array}{ll}
f & 0 \\
0 & f
\end{array}\right)
$$

We associate to this the complete intersection singularity $\left(X_{Q}, 0\right)$ given by

$$
\begin{aligned}
\mathbf{F}_{Q}(x, y, z, w) & =\left(F_{Q, 1}(x, y, z, w), F_{Q, 2}(x, y, z, w)\right) \\
& :=(-a(z, w)+x y, c(x, z, w)+y b(z, w)) .
\end{aligned}
$$

If $f=L_{y} F$, then we obtain back $\left(X_{Q}, 0\right)=(X, 0)$.

According to [14], the quadrangle ICIS correspond to matrix factorizations of the quadrangle hypersurface singularities $Q_{2,0}, S_{1,0}$, and $U_{1,0}$. They are given by nondegenerate invertible polynomials with $\left[G_{f}: G_{0}\right]=2$. In [13, Proposition 1], we classified such polynomials. The coordinates are chosen so that the action of $\widetilde{G}_{0}=$ 
Table 4 Functions $\mathbf{f}$ of 4 of the quadrangle hypersurface singularities

\begin{tabular}{lllll}
\hline Name & Type & $p_{1}, p_{2}, p_{3}$ & f & $a_{1}, a_{2}, a_{3}, a_{4}$ \\
\hline$Q_{2,0}$ & $\mathrm{IV}_{1}$ & $3,12,24$ & $a_{1} x^{3}+a_{2} x y^{4}+a_{3} y z^{2}+a_{4} x^{2} y^{2}$ & $1,1,1,-2$ \\
$S_{1,0}$ & $\mathrm{IV}_{2}$ & $5,10,20$ & $a_{1} x^{5}+a_{2} x y^{2}+a_{3} y z^{2}+a_{4} x^{3} y$ & $1,1,1,-2$ \\
$S_{1,0}^{\sharp}$ & $\mathrm{IV}_{2}^{\sharp}$ & $5,10,20$ & $a_{1} x^{2} z^{2}+a_{2} x y^{2}+a_{3} y z^{2}+a_{4} x^{3} y$ & $-1,1,1,-1$ \\
$U_{1,0}$ & $\mathrm{IV}_{2}^{\sharp}$ & $3,6,18$ & $a_{1} x z^{3}+a_{2} x y^{2}+a_{3} y z^{3}+a_{4} x^{2} y$ & $-1,1,1,-1$ \\
\hline
\end{tabular}

Table 5 Matrix factorizations of the functions $\mathbf{f}$

\begin{tabular}{llll}
\hline Name & Coord. change & f & Name \\
\hline$Q_{2,0}$ & $(x, y, z) \mapsto(w, x, z)$ & $x\left(a_{2} w x^{3}+a_{3} z^{2}+a_{4} x w^{2}\right)+\left(a_{1} w\right) \cdot w^{2}$ & $J_{2,0}^{\prime}$ \\
$S_{1,0}$ & $(x, y, z) \mapsto(x, z, w)$ & $x\left(a_{1} x^{4}+a_{2} z^{2}+a_{4} x^{2} z\right)+\left(a_{3} z\right) \cdot w^{2}$ & $K_{1,0}^{\prime}$ \\
$S_{1,0}$ & $(x, y, z) \mapsto(x, z, w)$ & $x\left(a_{1} x^{4}+a_{2} z^{2}+a_{4} x^{2} z\right)+\left(a_{3} w\right) \cdot z w$ & $L_{1,0}$ \\
$S_{1,0}^{\sharp}$ & $(x, y, z) \mapsto(x, z, w)$ & $x\left(a_{1} x w^{2}+a_{2} z^{2}+a_{4} x^{2} z\right)+\left(a_{3} z\right) \cdot w^{2}$ & $K_{1,0}^{b}$ \\
$S_{1,0}^{\#}$ & $(x, y, z) \mapsto(x, z, w)$ & $x\left(a_{1} x w^{2}+a_{2} z^{2}+a_{4} x^{2} z\right)+\left(a_{3} w\right) \cdot z w$ & $L_{1,0}^{\sharp}$ \\
$U_{1,0}$ & $(x, y, z) \mapsto(w, x, z)$ & $x\left(a_{2} x w+a_{3} z^{3}+a_{4} w^{2}\right)+\left(a_{1} z\right) \cdot z^{2} w$ & $M_{1,0}$ \\
$U_{1,0}$ & $(x, y, z) \mapsto(z, w, x)$ & $x\left(a_{1} x^{2} z+a_{3} x^{2} w\right)+\left(a_{2} w+a_{4} z\right) \cdot z w$ & $M_{1,0}^{\sharp}$ \\
$U_{1,0}$ & $(x, y, z) \mapsto(x, z, w)$ & $x\left(a_{1} w^{3}+a_{2} z^{2}+a_{4} x z\right)+\left(a_{3} z\right) \cdot w^{3}$ & $I_{1,0}$ \\
\hline
\end{tabular}

$\mathbb{Z} / 2 \mathbb{Z}$ on $\tilde{f}$ is given by $(x, y, z) \mapsto(-x,-y, z)$. The singularities $Q_{2,0}, S_{1,0}$, and $U_{1,0}$ are given by an invertible polynomial of type IV, namely

$$
\begin{aligned}
f(x, y, z) & =x^{p_{1}}+x y^{\frac{p_{2}}{p_{1}}}+y z^{\frac{p_{3}}{p_{2}}} \text { with }\left(p_{1}, p_{2}, p_{3}\right) \\
& =(3,12,24),(5,10,20), \text { and }(3,6,18)
\end{aligned}
$$

respectively. In [13, Proposition 2], we classified certain $4 \times 3$ matrices corresponding to data determined by such polynomials, see [13] for details. Using [13, Table 1, 9 and 10] and the notations used there, this amounts to the list of Table 4. The last column indicates the coefficients $a_{1}, a_{2}, a_{3}, a_{4} \in \mathbb{C}$ which are used in [13] and will be used in Sect. 4.

We now consider the matrix factorizations of the functions $\mathbf{f}$ of Table 4 . They are given in Table 5, where we use suitable coordinates $(x, z, w)$ instead of $(x, y, z)$.

In the case $Q_{2,0}$, the matrix factorization from [14, Table 2]

$$
q_{1}=\left(\begin{array}{cr}
y^{4}-\left(1+\lambda_{4}\right) x y^{2}+\lambda_{4} x^{2}-y \\
z^{2} \\
-x
\end{array}\right)
$$

is equivalent to the matrix factorization from Table 5 with $a_{1}=\lambda_{4}, a_{2}=-1, a_{3}=1$, $a_{4}=1+\lambda_{4}$, which is seen by adding the second column multiplied by $y^{3}-\left(1+\lambda_{4}\right) x y$ to the first column. 


\section{An Extension of the Berglund-Hübsch Duality}

We shall now show that the duality between the quadrangle complete intersection singularities can be derived from the Berglund-Hübsch transposition of certain polynomials in 4 variables. We use the procedure in [13] to associate a weighted homogeneous non-invertible polynomial with 4 terms in 4 variables to each of the quadrangle complete intersection singularities. We consider the complete intersection singularities associated to the matrix factorizations in Table 5 defined by equations $\left(F_{Q, 1}, F_{Q, 2}\right)$, where we set $a_{i}=1, i=1, \ldots, 4$, and where we take a suitable order of the terms. Moreover, in the equation for $I_{1,0}$ we replace $X Z+Y Z$ by $X^{2}+Y^{2}$. We also substitute temporarily the variables $x, y, z, w$ by capital letters $X, Y, Z, W$. We have the following 4 cases:

(a) $F_{Q, 1}(X, Y, Z, W)=X Y-W^{2}$,

(b) $F_{Q, 1}(X, Y, Z, W)=X Y-W^{3}$,

(c) $F_{Q, 1}(X, Y, Z, W)=X Y-Z W$,

(d) $F_{Q, 1}(X, Y, Z, W)=X Y-Z^{2} W$.

We make the following coordinate substitutions in $F_{Q, 2}(X, Y, Z, W)$ :

(a) $X Y-W^{2}: X:=x^{2} w, Y:=y^{2} w, Z:=z, W:=x y w$,

(b) $X Y-W^{3}: X:=x^{6} w^{3}, Y:=y^{6} w^{3}, Z:=z, W:=x^{2} y^{2} w^{2}$.

(c) $X Y-Z W: X:=x w, Y:=y z, Z:=x z, W:=y w$,

(d) $X Y-Z^{2} W: X:=y^{2} z^{2}, Y:=x^{2} w^{2}, Z:=x z, W:=y^{2} w^{2}$,

Then the polynomial $F_{Q, 2}(X, Y, Z, W)$ is transformed to a non-invertible polynomial

$$
f(x, y, z, w)=\sum_{i=1}^{4} x^{E_{i 1}} y^{E_{i 2}} z^{E_{i 3}} w^{E_{i 4}}
$$

for a $4 \times 4$-matrix $E$ of exponents. The corresponding polynomials are listed in Table 6 .

This procedure can be explained as follows. We observe that the kernel of the matrix $E$ is generated by one of the following vectors:

(a),(b) $(1,1,0,-2)^{\mathrm{T}}$,

(c),(d) $(1,1,-1,-1)^{\mathrm{T}}$.

Let $R:=\mathbb{C}[x, y, z, w]$. There exists a $\mathbb{Z}$-graded structure on $R$ given by the respective $\mathbb{C}^{*}$-action (here $\lambda \in \mathbb{C}^{*}$ ):

$$
\begin{aligned}
& \text { (a), (b) } \lambda *(x, y, z, w)=\left(\lambda x, \lambda y, z, \lambda^{-2} w\right) \\
& \text { (c), (d) } \lambda *(x, y, z, w)=\left(\lambda x, \lambda y, \lambda^{-1} z, \lambda^{-1} w\right)
\end{aligned}
$$

Let $R=\bigoplus_{i \in \mathbb{Z}} R_{i}$ be the decomposition of $R$ according to one of these $\mathbb{Z}$-gradings. The new coordinates $X, Y, Z, W$ are some invariant polynomials with respect to these actions and they satisfy the relation given by the corresponding first equation. 
Table 6 Strange duality

\begin{tabular}{llll}
\hline Name & $\left(F_{Q, 1}, F_{Q, 2}\right)$ & $f$ & Dual \\
\hline$J_{2,0}^{\prime}$ & $\left(X Y-W^{2}, X^{3} W+Y W+Z^{2}+X W^{2}\right)$ & $x^{7} y w^{4}+x y^{3} w^{2}+z^{2}+x^{4} y^{2} w^{3}$ & $J_{2,0}^{\prime}$ \\
$K_{1,0}^{\prime}$ & $\left(X Y-W^{2}, X^{4}+Z^{2}+Y Z+X^{2} Z\right)$ & $x^{8} w^{4}+z^{2}+y^{2} z w+x^{4} z w^{2}$ & $K_{1,0}^{\prime}$ \\
$K_{1,0}^{b}$ & $\left(X Y-W^{2}, X W^{2}+Z^{2}+Y Z+X^{2} Z\right)$ & $x^{4} y^{2} w^{3}+z^{2}+y^{2} z w+x^{4} z w^{2}$ & $L_{1,0}$ \\
$L_{1,0}$ & $\left(X Y-Z W, X^{4}+Z^{2}+Y W+X^{2} Z\right)$ & $x^{4} w^{4}+x^{2} z^{2}+y^{2} z w+x^{3} z w^{2}$ & $K_{1,0}^{b}$ \\
$L_{1,0}^{\sharp}$ & $\left(X Y-Z W, X W^{2}+Z^{2}+Y W+X^{2} Z\right)$ & $x y^{2} w^{3}+x^{2} z^{2}+y^{2} z w+x^{3} z w^{2}$ & $L_{1,0}^{\sharp}$ \\
$M_{1,0}$ & $\left(X Y-Z^{2} W, Z^{3}+W^{2}+Y Z+X W\right)$ & $x^{3} z^{3}+y^{4} w^{4}+x^{3} z w^{2}+y^{4} z^{2} w^{2}$ & $M_{1,0}$ \\
$M_{1,0}^{\sharp}$ & $\left(X Y-Z W, X^{2} W+Y Z+Y W+X^{2} Z\right)$ & $x^{2} y w^{3}+x y z^{2}+y^{2} z w+x^{3} z w^{2}$ & $M_{1,0}^{\sharp}$ \\
$I_{1,0}$ & $\left(X Y-W^{3}, X^{2}+Y^{2}+Z^{2}+W^{3}\right)$ & $x^{12} w^{6}+y^{12} w^{6}+z^{2}+x^{6} y^{6} w^{6}$ & $I_{1,0}$ \\
\hline
\end{tabular}

Table 7 Functions $\mathbf{h}$ of 4 of the quadrangle hypersurface singularities

\begin{tabular}{llll}
\hline Name & $\mathbf{f}$ & Coord. change & $\mathbf{h}$ \\
\hline$Q_{2,-1}$ & $w^{3}+x^{4} w+x z^{2}-2 x^{2} w^{2}$ & $w \mapsto w+x^{2}$ & $w^{3}+x^{2} w^{2}+x z^{2}-x^{3} z$ \\
$S_{1,-1}$ & $x^{5}+x z^{2}+z w^{2}-2 x^{3} z$ & $z \mapsto z+x^{2}$ & $x z^{2}+z w^{2}+x^{2} w^{2}-w x^{3}$ \\
$S_{1,-1}^{\sharp}$ & $-x^{2} w^{2}+x z^{2}+z w^{2}-x^{3} z$ & $z \mapsto z+x^{2}$ & $x z^{2}+x^{3} z+z w^{2}-x^{3} w$ \\
$U_{1,-1}$ & $-z^{3} w+x^{2} w+x z^{3}-x w^{2}$ & $x \mapsto x+w$ & $x^{2} w+x w^{2}+x z^{3}-z w^{2}$ \\
\hline
\end{tabular}

An inspection of Table 6 shows that the Berglund-Hübsch transpose of the polynomial $f$ is either the polynomial $f$ itself or another polynomial appearing in the table. This leads to the indicated duality.

\section{Virtual Isolated Complete Intersection Singularities}

We now derive the equations for the virtual singularities.

In [13, Section 4], we associated a polynomial $\mathbf{h}$ to $\mathbf{f}$, which defines the corresponding virtual bimodal hypersurface singularity. This is done as follows. We consider the polynomial $\mathbf{f}(x, z, w)$ from Table 4 (using the coordinate transformation of Table 5) with the choice of coefficients $a_{1}, a_{2}, a_{3}, a_{4}$ given in the last column. Then the corresponding equation defines a non-isolated singularity. We consider the cusp singularity

$$
\mathbf{f}(x, z, w)-x z w
$$

and perform the coordinate change indicated in Table 7.

Then this polynomial is transformed to

$$
\mathbf{h}(x, z, w)-x z w
$$


where the polynomial $\mathbf{h}(x, z, w)$ is indicated in Table 7 . The polynomial $\mathbf{h}(x, z, w)$ has an isolated singularity at the origin, but also an additional critical point of type $A_{1}$ outside the origin. Moreover, if we consider the 1-parameter family $\mathbf{h}(x, z, w)-t \cdot x z w$ for $t \in \mathbb{C}$, then, for $t \neq 0,1$, the polynomial $\mathbf{h}(x, z, w)-t \cdot x z w$ has two additional critical points of type $A_{1}$ outside the origin. One of them merges with the singularity of $\mathbf{h}(x, z, w)$ at the origin for $t=0$ and the other one merges with the singularity of $\mathbf{f}(x, z, w)-x z w$ at the origin for $t=1$.

Example 2 Consider the case $Q_{2,0}$. Then

$$
\begin{aligned}
& \mathbf{f}\left(x, z, w+x^{2}\right)-x z\left(w+x^{2}\right) \\
& \quad=\left(w+x^{2}\right)^{3}+x^{4}\left(w+x^{2}\right)+x z^{2}-2 x^{2}\left(w+x^{2}\right)^{2}-x z\left(w+x^{2}\right) \\
& \quad=w^{3}+x^{2} w^{2}+x z^{2}-x^{3} z-x z w \\
& \quad=\mathbf{h}(x, z, w)-x z w .
\end{aligned}
$$

The polynomial $\mathbf{h}(x . z, w)$ has a singularity of Arnold type $Q_{12}$ at the origin. On the other hand, for $t \neq 0$,

$$
\begin{aligned}
& \mathbf{h}\left(x, z, w-\frac{1}{t} x^{2}\right)-t \cdot x z\left(w-\frac{1}{t} x^{2}\right) \\
& =\left(w-\frac{1}{t} x^{2}\right)^{3}+x^{2}\left(w-\frac{1}{t} x^{2}\right)^{2}+x z^{2}-x^{3} z-t \cdot x z\left(w-\frac{1}{t} x^{2}\right) \\
& =w^{3}+\left(3 \frac{1}{t^{2}}-2 \frac{1}{t}\right) x^{4} w+\left(\frac{1}{t^{2}}-\frac{1}{t^{3}}\right) x^{6}+x z^{2}+\left(1-3 \frac{1}{t}\right) x^{2} w^{2}-t \cdot x z w .
\end{aligned}
$$

Using the proof of [12, Theorem 10], one can show that, for $t \neq 1$, this is a cusp singularity of type $T_{3,3,6}$. For $t=1$, it is a cusp singularity of type $T_{3,3,7}$. Using this, one can check the above statements.

Now we are looking at possible matrix factorizations of the polynomials $\mathbf{h}$ of Table 7. They are listed together with the corresponding isolated complete intersection singularities in Table 8 . The resulting pairs of polynomials $\left(\mathbf{h}_{1}, \mathbf{h}_{2}\right)$ are called the virtual quadrangle complete intersection singularities or, shortly, the virtual singularities and they are denoted by replacing the index 0 by -1 .

There is another matrix factorization in the case $U_{1,-1}$, namely

$$
x\left(x w+z^{3}+w^{2}\right)-z w \cdot w
$$

It is equivalent to the matrix factorization corresponding to $I_{1,-1}$.

The equations $\left(\mathbf{h}_{1}, \mathbf{h}_{2}\right)$ will be used in the sequel. Note that they differ from the equations in Table 2 in a similar way as the equations in [12, Table 12] for the interpretation of Arnold's strange duality as the Berglund-Hübsch transposition of invertible polynomials differ partly from Arnold's equations. In a similar way, the equations in [13] deviate from the equations of Arnold as well, cf. [13, Table 10]. 
Table 8 Virtual singularities

\begin{tabular}{llll}
\hline Name & Matrix factorization of $\mathbf{h}$ & $\left(\mathbf{h}_{1}, \mathbf{h}_{2}\right)$ & Virtual \\
\hline$Q_{2,-1}$ & $x\left(-x^{2} z+z^{2}+x w^{2}\right)+w \cdot w^{2}$ & $\left(x y-w^{2},-x^{2} z+y w+z^{2}+x w^{2}\right)$ & $J_{2,-1}^{\prime}$ \\
$S_{1,-1}$ & $x\left(-x^{2} w+z^{2}+x w^{2}\right)+z \cdot w^{2}$ & $\left(x y-w^{2},-x^{2} w+z^{2}+y z+x w^{2}\right)$ & $K_{1,-1}^{\prime}$ \\
$S_{1,-1}^{\sharp}$ & $x\left(-x^{2} w+z^{2}+x^{2} z\right)+z \cdot w^{2}$ & $\left(x y-w^{2},-x^{2} w+z^{2}+y z+x^{2} z\right)$ & $K_{1,-1}^{b}$ \\
$S_{1,-1}$ & $x\left(-x^{2} w+z^{2}+x w^{2}\right)+z w \cdot w$ & $\left(x y-z w,-x^{2} w+z^{2}+y w+x w^{2}\right)$ & $L_{1,-1}$ \\
$S_{1,-1}^{\sharp}$ & $x\left(-x^{2} w+z^{2}+x^{2} z\right)+z w \cdot w$ & $\left(x y-z w,-x^{2} w+z^{2}+y w+x^{2} z\right)$ & $L_{1,-1}^{\sharp}$ \\
$U_{1,-1}$ & $x\left(x w+z^{3}+w^{2}\right)-z \cdot w^{2}$ & $\left(x y-w^{2},-y z+x w+z^{3}+w^{2}\right)$ & $M_{1,-1}$ \\
$U_{1,-1}$ & $x\left(-z^{2}+x^{2} w\right)+z w \cdot(z+w)$ & $\left(x y-z w,-z^{2}+y w+x^{2} w+y z\right)$ & $M_{1,-1}^{\sharp}$ \\
$U_{1,-1}$ & $x\left(-x w+z^{2}+x z\right)+z \cdot w^{3}$ & $\left(x y-w^{3},-x w+z^{2}+y z+x z\right)$ & $I_{1,-1}$ \\
\hline
\end{tabular}

Let $\left(\mathbf{h}_{1}, \mathbf{h}_{2}\right)$ with

$$
\mathbf{h}_{1}(x, y, z, w)=x y-z^{c} w^{d}, \quad \mathbf{h}_{2}(x, y, z, w)=\sum_{i=1}^{4} a_{i} x^{A_{i 1}} y^{A_{i 2}} z^{A_{i 3}} w^{A_{i 4}}
$$

be a virtual singularity. We consider the polynomial $\mathbf{h}_{2}(x, y, z, w)$ on the zero set of the polynomial $\mathbf{h}_{1}(x, y, z, w)$. This means that we substitute $y=x^{-1} z^{c} w^{d}$ in the polynomial $\mathbf{h}_{2}(x, y, z, w)$ to get a Laurent polynomial

$$
\mathbf{h}_{2}^{\prime}(x, z, w):=\mathbf{h}_{2}\left(x, x^{-1} z^{c} w^{d}, z, w\right)=\sum_{i=1}^{4} a_{i} x^{A_{i 1}-A_{i 2}} z^{A_{i 3}+c A_{i 2}} w^{A_{i 4}+d A_{i 2}}
$$

in the variables $x, z, w$. Define $\operatorname{Supp}\left(\mathbf{h}_{2}^{\prime}\right):=\left\{\left(A_{i 1}-A_{i 2}, A_{i 3}+c A_{i 2}, A_{i 4}+d A_{i 2}\right) \in\right.$ $\left.\mathbb{Z}^{3} \mid i=1, \ldots, 4\right\}$. Let $\Gamma_{\infty}\left(\mathbf{h}_{2}^{\prime}\right)$ be the Newton polyhedron of $\mathbf{h}_{2}^{\prime}$ at infinity [17], i.e. $\Gamma_{\infty}\left(\mathbf{h}_{2}^{\prime}\right)$ is the convex closure in $\mathbb{R}^{3}$ of $\operatorname{Supp}\left(\mathbf{h}_{2}^{\prime}\right) \cup\{0\}$. The Newton polyhedron $\Gamma_{\infty}\left(\mathbf{h}_{2}^{\prime}\right)$ has two two-dimensional faces which do not contain the origin. Call these faces $\Sigma_{1}$ and $\Sigma_{2}$. Let $I_{k}:=\left\{i \in\{1, \ldots, 4\} \mid\left(A_{i 1}-A_{i 2}, A_{i 3}+c A_{i 2}, A_{i 4}+d A_{i 2}\right) \in \Sigma_{k}\right\}$, $k=1,2$, and let

$$
\mathbf{h}_{2, k}=\sum_{i \in I_{k}} a_{i} x^{A_{i 1}} y^{A_{i 2}} z^{A_{i 3}} w^{A_{i 4}}
$$

Then $\left(\mathbf{h}_{1}, \mathbf{h}_{2, k}\right)$ defines a non-isolated weighted homogeneous complete intersection singularity. The polynomials $\mathbf{h}_{2,1}$ and $\mathbf{h}_{2,2}$ and their systems of weights are listed in Table 9.

\section{Dolgachev Numbers}

We shall now define Dolgachev numbers for our virtual singularities. 
Table 9 Decomposition of equations

\begin{tabular}{lllll}
\hline Name & $\mathbf{h}_{2,1}$ & Weights & $\mathbf{h}_{2,2}$ & Weights \\
\hline$J_{2,-1}^{\prime}$ & $-x^{2} z+z^{2}+x w^{2}$ & $2,4,4,3 ; 6,8$ & $z^{2}+x w^{2}+y w$ & $2,6,5,4 ; 8,10$ \\
$K_{1,-1}^{\prime}$ & $-x^{2} w+z^{2}+x w^{2}$ & $2,2,3,2 ; 4,6$ & $z^{2}+x w^{2}+y z$ & $2,4,4,3 ; 6,8$ \\
$K_{1,-1}^{b}$ & $-x^{2} w+x^{2} z+y z$ & $2,4,3,3 ; 6,7$ & $x^{2} z+y z+z^{2}$ & $2,4,4,3 ; 6,8$ \\
$L_{1,-1}$ & $-x^{2} w+z^{2}+x w^{2}$ & $2,3,3,2 ; 5,6$ & $z^{2}+x w^{2}+y w$ & $2,5,4,3 ; 7,8$ \\
$L_{1,-1}^{\sharp}$ & $-x^{2} w+x^{2} z+y w$ & $2,4,3,3 ; 6,7$ & $x^{2} z+y w+z^{2}$ & $2,5,4,3 ; 7,8$ \\
$M_{1,-1}$ & $x w+z^{3}+w^{2}$ & $3,3,2,3 ; 6,6$ & $z^{3}+w^{2}-y z$ & $2,4,2,3 ; 6,6$ \\
$M_{1,-1}^{\sharp}$ & $-z^{2}+x^{2} w+y z$ & $2,3,3,2 ; 5,6$ & $x^{2} w+y z+y w$ & $2,4,3,3 ; 6,7$ \\
$I_{1,-1}$ & $-x w+y z+x z$ & $3,3,2,2 ; 6,5$ & $y z+x z+z^{2}$ & $3,3,3,2 ; 6,6$ \\
\hline
\end{tabular}

The Dolgachev numbers of the virtual singularity $\left(\mathbf{h}_{1}, \mathbf{h}_{2}\right)$ are defined in a similar way as [13, Section 5]. Let $i=1,2$ and let $V_{i}:=\left\{(x, y, z, w) \in \mathbb{C}^{4} \mid \mathbf{h}_{1}(x, y, z, w)=\right.$ $\left.0, \mathbf{h}_{2, i}(x, y, z, w)=0\right\}$. We consider the $\mathbb{C}^{*}$-action on $V_{i}$ given by the system of weights of $\left(\mathbf{h}_{1}, \mathbf{h}_{2, i}\right)$ (see Table 9 ). We consider exceptional orbits (i.e. orbits with a non-trivial isotropy group) of this action. We distinguish between three cases:

(A) $V_{i}$ contains a linear subspace $L$ of $\mathbb{C}^{4}$ of codimension 2 obtained by setting two coordinates to be zero.

(B) $V_{i}=U \cup U^{\prime}$, where

$$
\begin{aligned}
& \left(\mathbf{h}_{1}(x, y, z, w), \mathbf{h}_{2, i}(x, y, z, w)\right)=\left(g_{1}(x, y, w), z g_{2}(x, y, z)\right), \\
& U=\left\{(x, y, z, w) \in \mathbb{C}^{4} \mid g_{1}(x, y, w)=z=0\right\}, \\
& U^{\prime}=\left\{(x, y, z, w) \in \mathbb{C}^{4} \mid g_{1}(x, y, w)=g_{2}(x, y, z)=0\right\} .
\end{aligned}
$$

(C) $V_{i}$ is not of the form of (A) or (B).

In case (A) we consider those exceptional orbits which are not contained in $L$. In case (B) we consider those exceptional orbits which are not contained in $U$. In case (C) we consider those exceptional orbits which do not coincide with the singular locus of $V_{i}$. We call these the principal orbits. It turns out that in all cases we have exactly two principal orbits.

Definition The Dolgachev numbers of the virtual singularity $\left(\mathbf{h}_{1}, \mathbf{h}_{2}\right)$ are the numbers $\alpha_{1}, \alpha_{2} ; \alpha_{3}, \alpha_{4}$ where $\alpha_{1}, \alpha_{2}$ and $\alpha_{3}, \alpha_{4}$ are the orders of the isotropy groups of the principal exceptional orbits of $\left(\mathbf{h}_{1}, \mathbf{h}_{2,1}\right)$ and $\left(\mathbf{h}_{1}, \mathbf{h}_{2,2}\right)$ respectively.

The Dolgachev numbers of the virtual singularities are computed as follows. The two pairs of polynomials $\left(\mathbf{h}_{1}, \mathbf{h}_{2, i}\right), i=1,2$, of Table 9 define non-isolated weighted homogeneous complete intersection singularities of certain types. The systems of weights correspond to the five quadrangle ICIS and three elliptic complete intersection singularities as considered by Wall [23]. We indicate the notation of Wall [23] in Table 10. The corresponding orbifold curves have genus zero. We list the orders of the 
isotropy groups of the exceptional orbits of these ICIS in this table (see also [8]). Some of them correspond to the orders of the isotropy groups of the exceptional orbits for the non-isolated singularities given by the pairs $\left(\mathbf{h}_{1}, \mathbf{h}_{2, i}\right), i=1,2$. Those ones which do not occur are stroken out. The orders of the isotropy groups of the principal orbits are indicated in bold face. We also indicate for each pair which of the corresponding cases (A), (B), or (C) applies. An exceptional orbit which coincides with the singular locus is marked by *. The Dolgachev numbers $\alpha_{1}, \alpha_{2} ; \alpha_{3}, \alpha_{4}$ of the virtual singularities are indicated in the last column.

Example 3 (a) We consider the singularity $K_{1,-1}^{b}$. We have $\left(\mathbf{h}_{1}, \mathbf{h}_{2,1}\right)=(x y-$ $\left.w^{2},-x^{2} w+x^{2} z+y z\right)$ with the system of weights $(2,4,3,3 ; 6,7)$. We are in case (C). The exceptional orbits are

$$
\begin{aligned}
x=y=w=0 & \text { singular line, order of isotropy group: } 3 \\
y=z=w=0 & \text { order of isotropy group: } 2 \\
x=z=w=0 & \text { order of isotropy group: } 4
\end{aligned}
$$

This gives $\left(\alpha_{1}, \alpha_{2}\right)=(2,4)$.

(b) We again consider the singularity $K_{1,-1}^{b}$, but now $\left(\mathbf{h}_{1}, \mathbf{h}_{2,2}\right)=\left(x y-w^{2}, x^{2} z+\right.$ $\left.y z+z^{2}\right)$ with the system of weights $(2,4,4,3 ; 6,8)$. Here we are in case (B). The singular locus is the curve $z=x^{2}+y=x^{3}+w^{2}=0$ with trivial isotropy group. It is contained in $U=\left\{x y-w^{2}=z=0\right\}$. The exceptional orbits contained in $U$ are

$$
\begin{array}{ll}
y=z=w=0 & \text { order of isotropy group: } 2 \\
x=z=w=0 & \text { order of isotropy group: } 4
\end{array}
$$

The exceptional orbits not contained in $U$ are

$$
\begin{array}{cc}
y=w=x^{2}+z=0 & \text { order of isotropy group: } 2 \\
x=w=y+z=0 & \text { order of isotropy group: } 4
\end{array}
$$

This gives $\left(\alpha_{3}, \alpha_{4}\right)=(2,4)$.

(c) We consider the case $L_{1,-1}$ with $\left(\mathbf{h}_{1}, \mathbf{h}_{2,1}\right)=\left(x y-z w,-x^{2} w+z^{2}+x w^{2}\right)$. The system of weights is $(2,3,3,2 ; 5,6)$. Here $V_{1}$ contains the hyperplane $L=\{x=$ $z=0\}$, so we are in case (A). The exceptional orbits contained in $L$ are

$$
\begin{gathered}
x=z=w=0 \quad \text { singular line, order of isotropy group: } 3 \\
x=y=z=0 \quad \text { order of isotropy group: } 2
\end{gathered}
$$

The exceptional orbits not contained in $L$ are

$$
\begin{array}{cc}
y=z=w=0 & \text { order of isotropy group: } 2 \\
x-w=y=z=0 & \text { order of isotropy group: } 2
\end{array}
$$

This gives $\left(\alpha_{1}, \alpha_{2}\right)=(2,2)$.

Remark 4 Using the primary decomposition algorithm of the computer algebra software SingulaR [5], one can show that, for each pair $\left(\mathbf{h}_{1}, \mathbf{h}_{2, i}\right)$ where we have case (A), the subspace $L$ is an irreducible component of $V_{i}$. If one removes this component $L$ in case (A), the component $U$ in case (B), and the point corresponding to the singular line in case $(\mathrm{C})$, one gets $\mathbb{P}_{\alpha_{2 i-1}, \alpha_{2 i}}^{1}$ with one point removed. Here $\mathbb{P}_{\alpha_{2 i-1}, \alpha_{2 i}}^{1}$ denotes the complex projective line with two orbifold points with singularities $\mathbb{Z} / \alpha_{j} \mathbb{Z}$, $j=2 i-1,2 i$. 


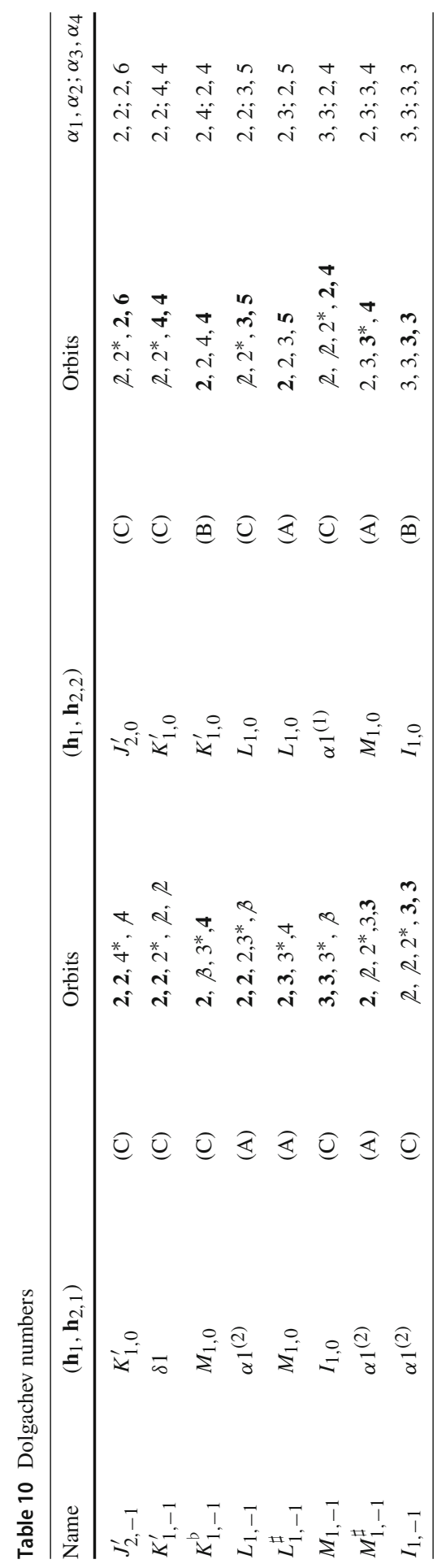




\section{Gabrielov Numbers}

We now want to define Gabrielov numbers. They will be defined as in [13, Section 5]. For this purpose, we consider the pairs $\left(\mathbf{h}_{1}, \mathbf{h}_{2}\right)$ of polynomials of Table 8. Here the first three cases $J_{2,-1}^{\prime}, K_{1,-1}^{\prime}$, and $K_{1,-1}^{b}$ are suspensions of the curve singularities $J_{2,-1}$, $K_{1,-1}$, and $K_{1,-1}^{\sharp}$. In these cases, we consider as the first polynomial $\mathbf{h}_{1}(x, y, z, w):=$ $x y-w^{2}-z^{2}$. Then we consider the complete intersection singularity $\left(X^{\prime}, 0\right)$ defined by

$$
\left\{\begin{array}{l}
\mathbf{h}_{1}(x, y, z, w), \\
\mathbf{h}_{2}(x, y, z, w)-z w .
\end{array}\right.
$$

As in [12], one can show that the singularity $\left(X^{\prime}, 0\right)$ is $\mathcal{K}$-equivalent to the singularity defined by

$$
\left\{\begin{array}{l}
x y-z^{\gamma_{1}}-w^{\gamma_{2}} \\
x^{\gamma_{3}}+y^{\gamma_{4}}-z w
\end{array} .\right.
$$

This means that $\left(X^{\prime}, 0\right)$ is a cusp singularity of type $T_{\gamma_{1}, \gamma_{3}, \gamma_{2}, \gamma_{4}}^{2}$ in the notation of [6, 3.1].

Definition The Gabrielov numbers of the virtual singularity given by the pair $\left(\mathbf{h}_{1}, \mathbf{h}_{2}\right)$ are the numbers $\gamma_{1}, \gamma_{2} ; \gamma_{3}, \gamma_{4}$.

Proposition 5 The Gabrielov numbers of the virtual quadrangle complete intersection singularities are given by Table 11.

Proof We consider Wall's reduction of the virtual quadrangle singularities according to Table 8 . The cusp singularity $\mathbf{H}^{\prime}:=\left(\mathbf{h}_{1}(x, y, z, w), \mathbf{h}_{2}(x, y, z, w)-z w\right)$ corresponds to the hypersurface cusp singularity $L_{y} \mathbf{H}^{\prime}=\mathbf{h}(x, z, w)-x z w$. In all cases except $I_{1,-1}$, by transformations similar to the transformations in [13], we obtain the indicated Gabrielov numbers. More precisely, for a suitable permutation $\sigma:\{1,2,3,4\} \rightarrow$ $\{1,2,3,4\}$, the Gabrielov numbers satisfy $\left(\gamma_{\sigma(1)}, \gamma_{\sigma(2)}, \gamma_{\sigma(3)}, \gamma_{\sigma(4)}\right)=\left(2, \tilde{\gamma}_{1}-1, \tilde{\gamma}_{2}-\right.$ $\left.1, \tilde{\gamma}_{3}-1\right)$, where $\left(\tilde{\gamma}_{1}, \tilde{\gamma}_{2}, \tilde{\gamma}_{3}\right)$ are the Gabrielov numbers of the corresponding virtual hypersurface singularity.

In the case $I_{1,-1}$, we indicate the claimed $\mathcal{K}$-equivalence. We add the first polynomial $\mathbf{h}_{1}(x, y, z, w)$ to the second one $\mathbf{h}_{2}(x ; y, z, w)-z w$ and obtain

$$
\left(x y-w^{3}, x y-w^{3}-x w+z^{2}+y z+x z-z w\right) .
$$

By the transformation $w \mapsto w+y$, this is transformed to

$$
\left(x y-w^{3}-p_{1}(y, w),-y^{3}-w^{3}-q_{1}(y, w)-x w+z^{2}+x z-z w\right),
$$

where $p_{1}(y, w)$ and $q_{1}(y, w)$ are certain polynomials of degree 3 in the variables $y$ and $w$. Using [1, Lemma 7.3] and the fact that $y$ divides $p_{1}(y, w)$, one can get rid of 
the polynomial $p_{1}(y, w)$ with the help of the term $x y$. Similarly, one can get rid of the polynomial $q_{1}(y, w)$ in the second equation with the help of the term $z w$. Now we apply the transformation $w \mapsto w+x$. Then the pair (6.2) gets

$$
\left(x y-w^{3}-x^{3}-p_{2}(x, w),-x^{3}-y^{3}-q_{2}(x, w)-x w-x^{2}+z^{2}-z w\right),
$$

where $p_{2}(x, w)$ and $q_{2}(x, w)$ are again polynomials which can be removed. Applying the transformation $x \mapsto x+z$, one gets

$$
\left(x y-w^{3}-z^{3}-p_{3}(x, z),-x^{3}-y^{3}-q_{3}(x, z)-x w-x^{2}-2 x z-2 z w\right),
$$

again with certain removable polynomials $p_{3}(x, z)$ and $q_{3}(x, z)$. Finally, by the transformation $z \mapsto \frac{1}{2}(z-x)$ followed by $w \mapsto w-x$ and rescaling, we obtain

$$
\left(x y-z^{3}-w^{3}+p_{4}(x, w), x^{3}+y^{3}-z w\right),
$$

again with a removable polynomial $p_{4}(x, w)$.

By [6], one can compute Coxeter-Dynkin diagrams of the (global) singularities defined by $\left(\mathbf{h}_{1}, \mathbf{h}_{2}\right)$. In the cases $M_{1,-1}$ and $I_{1,-1}$, the polynomials $\mathbf{h}_{1}$ and $\mathbf{h}_{2}$ have to be interchanged. Let $X^{(1)}:=\left\{(x, y, z, w) \in \mathbb{C}^{4} \mid \mathbf{h}_{1}(x, y, z, w)=0\right\}$ and consider the function $\mathbf{h}_{2}: X^{(1)} \rightarrow \mathbb{C}$. It has besides the origin one or two additional critical points which are of type $A_{1}$. The singularity at the origin is indicated in Table $11 \mathrm{using}$ the notation of [21]. We define the Milnor number of the virtual singularity by the sum of the Milnor numbers of the singular points. It is equal to 12 in all cases.

One can compute that there exists a (strongly) distinguished basis of thimbles $\left(e_{1}, \ldots, e_{\mu+1}\right)=\left(e_{j}^{r} \mid 1 \leq j \leq 8,1 \leq r \leq M_{j}\right)$, where the intersection matrix of $\left(e_{1}, \ldots, e_{8}\right)=\left(e_{1}^{1}, \ldots, e_{8}^{1}\right)$ coincides with the intersection matrix of the system $\left(\widehat{\delta}_{1}^{\prime}, \ldots, \widehat{\delta}_{8}^{\prime}\right)$ of $\left[6\right.$, Sect. 2.3], the numbers $M_{1}, M_{3}, M_{8}$ are equal to one and the other numbers $M_{2}, M_{4}, M_{5}, M_{6}, M_{7}$ are indicated in Table 11, and the intersection matrix of $\left(e_{j}^{r} \mid 1 \leq j \leq 8,1 \leq r \leq M_{j}\right)$ is computed according to [6, Theorem 2.2.3]. By the proof of [6, Proposition 3.6.1], one can transform these bases to (strongly) distinguished bases of thimbles with Coxeter-Dynkin diagrams of the form $\Pi_{\gamma_{1}, \gamma_{2}, \gamma_{3}, \gamma_{4}}$ of Fig. 1, where $\gamma_{1}, \gamma_{2} ; \gamma_{3}, \gamma_{4}$ are the Gabrielov numbers of the virtual singularity.

\section{Strange Duality}

We now consider the duality defined in Sect. 1. We summarize the results on the Dolgachev and Gabrielov numbers of the virtual singularities in Table 3. From this table, we get the following result:

Theorem 6 The Gabrielov numbers of a virtual quadrangle complete intersection singularity coincide with the Dolgachev numbers of the dual one, and vice versa. 


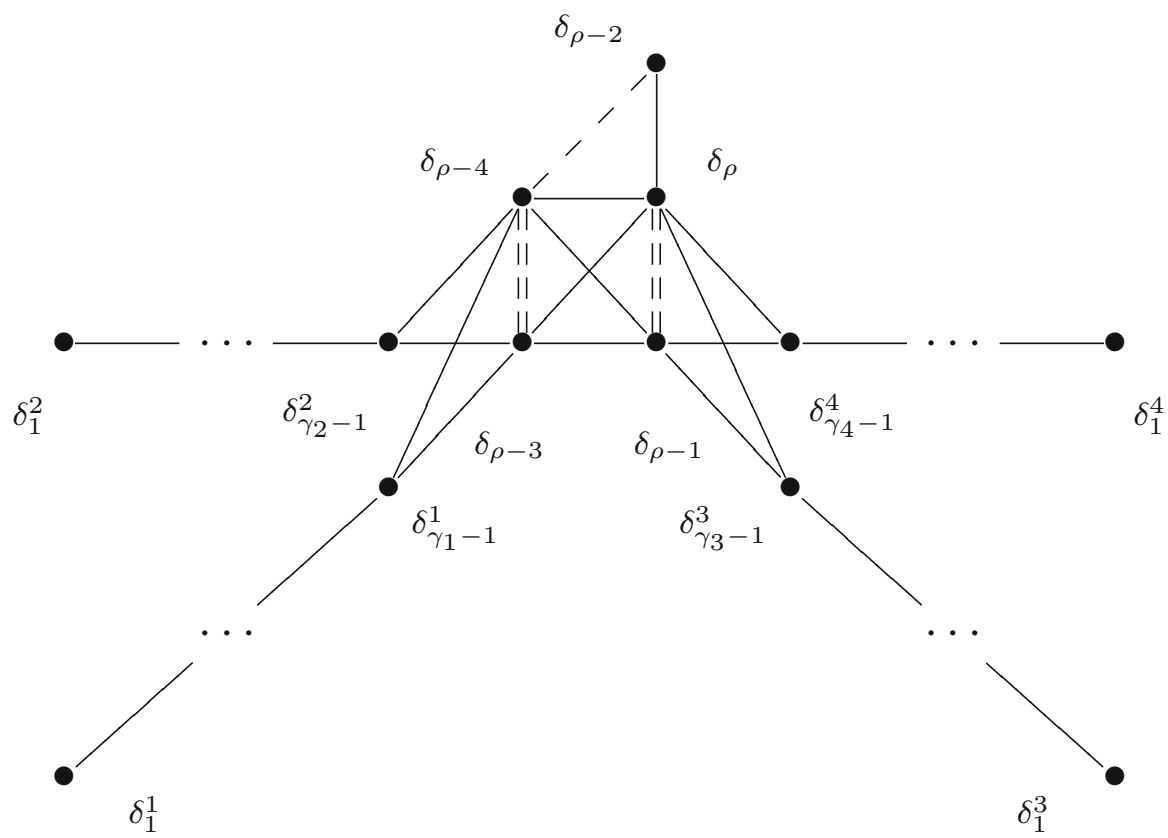

Fig. 1 The graph $\Pi_{\gamma_{1}, \gamma_{2}, \gamma_{3}, \gamma_{4}}$

Table 11 Coxeter-Dynkin diagrams of virtual singularities

\begin{tabular}{llllllll}
\hline Virtual & Germ at 0 & $M_{2}$ & $M_{4}$ & $M_{5}$ & $M_{6}$ & $M_{7}$ & $\gamma_{1}, \gamma_{2} ; \gamma_{3}, \gamma_{4}$ \\
\hline$J_{2,-1}^{\prime}$ & $J_{11}^{\prime}$ & 3 & 1 & 1 & 1 & $3+1$ & 2,$2 ; 2,5+1$ \\
$K_{1,-1}^{\prime}$ & $K_{10}^{\prime}$ & 2 & $2+1$ & $2+1$ & 1 & 1 & 2,$2 ; 3+1,3+1$ \\
$K_{1,-1}^{b}$ & $K_{11}^{\prime}$ & $2+1$ & 2 & 3 & 1 & 1 & 2,$2 ; 3,4+1$ \\
$L_{1,-1}$ & $L_{10}$ & 2 & $2+1$ & 1 & 1 & $2+1$ & $2,3+1 ; 2,3+1$ \\
$L_{1,-1}^{\sharp}$ & $L_{11}$ & $2+1$ & 3 & 1 & 1 & 2 & 2,$3 ; 2,4+1$ \\
$M_{1,-1}$ & $J_{10}^{\prime}$ & 3 & $1+1$ & $1+1$ & 1 & 2 & $2+1,2+1 ; 2,4$ \\
$M_{1,-1}^{\sharp}$ & $M_{11}$ & 2 & $2+1$ & 2 & 1 & 2 & 2,$3 ; 3,3+1$ \\
$I_{1,-1}$ & $M_{11}$ & 2 & 2 & 2 & $1+1$ & 2 & $2+1,3 ; 3,3$ \\
\hline
\end{tabular}

For another feature of this duality, we have to introduce some notions.

Let $f_{1}, \ldots, f_{k}$ be weighted homogeneous functions on $\mathbb{C}^{n}$ of degrees $d_{1}, \ldots, d_{k}$ with respect to weights $w_{1}, \ldots, w_{n}$. We suppose that the equations $f_{1}=f_{2}=\ldots=f_{k}=0$ define a complete intersection $X$ in $\mathbb{C}^{n}$. There is a natural $\mathbb{C}^{*}$-action on the space $\mathbb{C}^{n}$ defined by $\lambda *\left(x_{1}, \ldots, x_{n}\right)=\left(\lambda^{w_{1}} x_{1}, \ldots, \lambda^{w_{n}} x_{n}\right), \lambda \in \mathbb{C}^{*}$.

Let $A=\mathbb{C}\left[x_{1}, \ldots, x_{n}\right] /\left(f_{1}, \ldots, f_{k}\right)$ be the coordinate ring of $X$. Then the $\mathbb{C}^{*}$ action on $\mathbb{C}^{n}$ induces a natural grading $A=\oplus_{s=0}^{\infty} A_{s}$ on the ring $A$, where

$$
A_{s}:=\left\{g \in A \mid g\left(\lambda *\left(x_{1}, \ldots, x_{n}\right)\right)=\lambda^{s} g\left(x_{1}, \ldots, x_{n}\right) \text { for } \lambda \in \mathbb{C}^{*}\right\}
$$


We shall consider the Poincaré series $P_{X}(t)=\sum_{s=0}^{\infty} \operatorname{dim} A_{s} \cdot t^{s}$ of this graded algebra. One has

$$
P_{X}(t)=\frac{\prod_{j=1}^{k}\left(1-t^{d_{j}}\right)}{\prod_{i=1}^{n}\left(1-t^{w_{i}}\right)}
$$

For a map $\varphi: Z \rightarrow Z$ of a topological space $Z$, the zeta function is defined to be

$$
\zeta_{\varphi}(t)=\prod_{p \geq 0}\left\{\operatorname{det}\left(\mathrm{id}-\left.t \cdot \varphi_{*}\right|_{H_{p}(Z ; \mathbb{C})}\right)\right\}^{(-1)^{p}} .
$$

If, in the definition, we use the actions of the operators $\varphi_{*}$ on the reduced homology groups $\bar{H}_{p}(Z ; \mathbb{Z})$, we get the reduced zeta function

$$
\bar{\zeta}_{\varphi}(t)=\frac{\zeta_{\varphi}(t)}{(1-t)}
$$

For $0 \leq j \leq k$, let $X^{(j)}$ be the complete intersection given by the equations $f_{1}=\ldots=f_{j}=0\left(X^{(0)}=\mathbb{C}^{n}, X^{(k)}=X\right)$. The restriction of the function $f_{j}(j=$ $1, \ldots, k)$ to the variety $X^{(j-1)}$ defines a locally trivial fibration $X^{(j-1)} \backslash X^{(j)} \rightarrow \mathbb{C}^{*}$. Let $V^{(j)}=f_{j}^{-1}(1) \cap X^{(j-1)}$ be the typical fibre (Milnor fibre) of this fibration. Note that it is not necessarily smooth. There is a monodromy transformation $\varphi^{(j)}: V^{(j)} \rightarrow$ $V^{(j)}$ on it. Let

$$
\bar{\zeta}_{X, j}(t):=\bar{\zeta}_{\varphi(j)}(t)
$$

One can show that $\left(\varphi_{*}^{(j)}\right)^{d_{j}}=$ id and therefore $\bar{\zeta}_{X, j}(t)$ can be written in the form

$$
\prod_{\ell \mid d_{j}}\left(1-t^{\ell}\right)^{\alpha_{\ell}}, \alpha_{\ell} \in \mathbb{Z}
$$

Following Saito $[18,19]$, we define the Saito dual to $\bar{\zeta}_{X, j}(t)$ to be the rational function

$$
\bar{\zeta}_{X, j}^{*}(t)=\prod_{m \mid d_{j}}\left(1-t^{m}\right)^{-\alpha_{\left(d_{j} / m\right)}}
$$

(note that different degrees $d_{j}$ are used for different $j$ ).

Let $Y^{(k)}=\left(X^{(k)} \backslash\{0\}\right) / \mathbb{C}^{*}$ be the space of orbits of the $\mathbb{C}^{*}$-action on $X^{(k)} \backslash\{0\}$ and $Y_{m}^{(k)}$ be the set of orbits for which the isotropy group is the cyclic group of order $m$. For a topological space $Z$, denote by $\chi(Z)$ its Euler characteristic. Define

$$
\operatorname{Or}_{X}(t):=\prod_{m \geq 1}\left(1-t^{m}\right)^{\chi\left(Y_{m}^{(k)}\right)}
$$


Now let $X$ be a complete intersection in $\mathbb{C}^{4}$ defined by two polynomial equations $f_{1}=f_{2}=0$ and assume that both $X^{(1)}=f_{1}^{-1}(0)$ and $X^{(2)}=X$ have isolated singularities at the origin. Moreover, assume that $X^{(1)}$ has a singularity of type $A_{1}$. Consider the mapping $F:=\left(f_{1}, f_{2}\right): \mathbb{C}^{4} \rightarrow \mathbb{C}^{2}$. Let $C_{F}$ be the critical locus of $F$ and $D_{F}=F\left(C_{F}\right)$. The mapping $\left.F\right|_{\mathbb{C}^{4}-F^{-1}\left(D_{F}\right)}: \mathbb{C}^{4}-F^{-1}\left(D_{F}\right) \rightarrow \mathbb{C}^{2}-D_{F}$ defines a locally trivial fibration. Assume that $(1,1) \notin D_{F}$. Let $V^{(1)}=f_{1}^{-1}(1)$ and $V_{2}=f_{2}^{-1}(1) \cap V^{(1)}$. (Note that $V_{2} \neq V^{(2)}$ but $V_{2}$ and $V^{(2)}$ are homeomorphic to each other.) Then $V_{2} \subset V^{(1)}$ and the monodromy transformation $\varphi^{(1)}: V^{(1)} \rightarrow V^{(1)}$ induces a relative monodromy operator $\widehat{\varphi}_{*}: H_{3}\left(V^{(1)}, V_{2} ; \mathbb{Z}\right) \rightarrow H_{3}\left(V^{(1)}, V_{2} ; \mathbb{Z}\right)$. Let

$$
\Delta_{X}(t):=\operatorname{det}\left(\mathrm{id}-\left.t \cdot \widehat{\varphi}_{*}\right|_{H_{3}\left(V^{(1)}, V_{2} ; \mathbb{C}\right)}\right)
$$

be the characteristic polynomial of this operator.

Proposition 7 We have

$$
\Delta_{X}(t)=(1-t)^{2} \prod_{j=1}^{2} \bar{\zeta}_{X, j}(t)
$$

Proof We have the following commutative diagram of split short exact sequences:

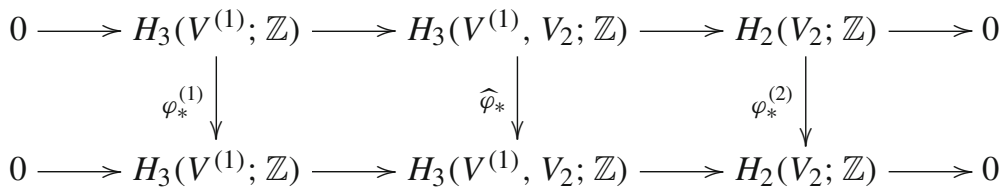

This shows that

$$
\begin{aligned}
\Delta_{X}(t) & =\operatorname{det}\left(\mathrm{id}-\left.t \cdot \varphi_{*}^{(1)}\right|_{H_{3}\left(V^{(1)} ; \mathbb{C}\right)}\right) \operatorname{det}\left(\mathrm{id}-\left.t \cdot \varphi_{*}^{(2)}\right|_{H_{2}\left(V_{2} ; \mathbb{C}\right)}\right) \\
& =\bar{\zeta}_{X, 1}(t)^{-1} \bar{\zeta}_{X, 2}(t)=(1-t)^{2} \prod_{j=1}^{2} \bar{\zeta}_{X, j}(t)
\end{aligned}
$$

since $\bar{\zeta}_{X, 1}(t)=(1-t)^{-1}$.

Let $X$ be an ICIS with a Coxeter-Dynkin diagram of type $\Pi_{\gamma_{1}, \gamma_{2}, \gamma_{3}, \gamma_{4}}$. Then the polynomial $\Delta_{X}(t)$ is equal to the characteristic polynomial $\Delta\left(\Pi_{\gamma_{1}, \gamma_{2}, \gamma_{3}, \gamma_{4}}\right)(t)$ of the Coxeter element corresponding to this Coxeter-Dynkin diagram. By [6, Proposition 3.6.2], we have

$$
\Delta\left(\Pi_{\gamma_{1}, \gamma_{2}, \gamma_{3}, \gamma_{4}}\right)(t)=(1-t)^{2} \Delta\left(S_{\gamma_{1}, \gamma_{2}, \gamma_{3}, \gamma_{4}}\right)(t)
$$

where $\Delta\left(S_{\gamma_{1}, \gamma_{2}, \gamma_{3}, \gamma_{4}}\right)(t)$ is the characteristic polynomial of the Coxeter element corresponding to the graph $S_{\gamma_{1}, \gamma_{2}, \gamma_{3}, \gamma_{4}}$ depicted in Fig. 2. (Note that there is a slight mistake 


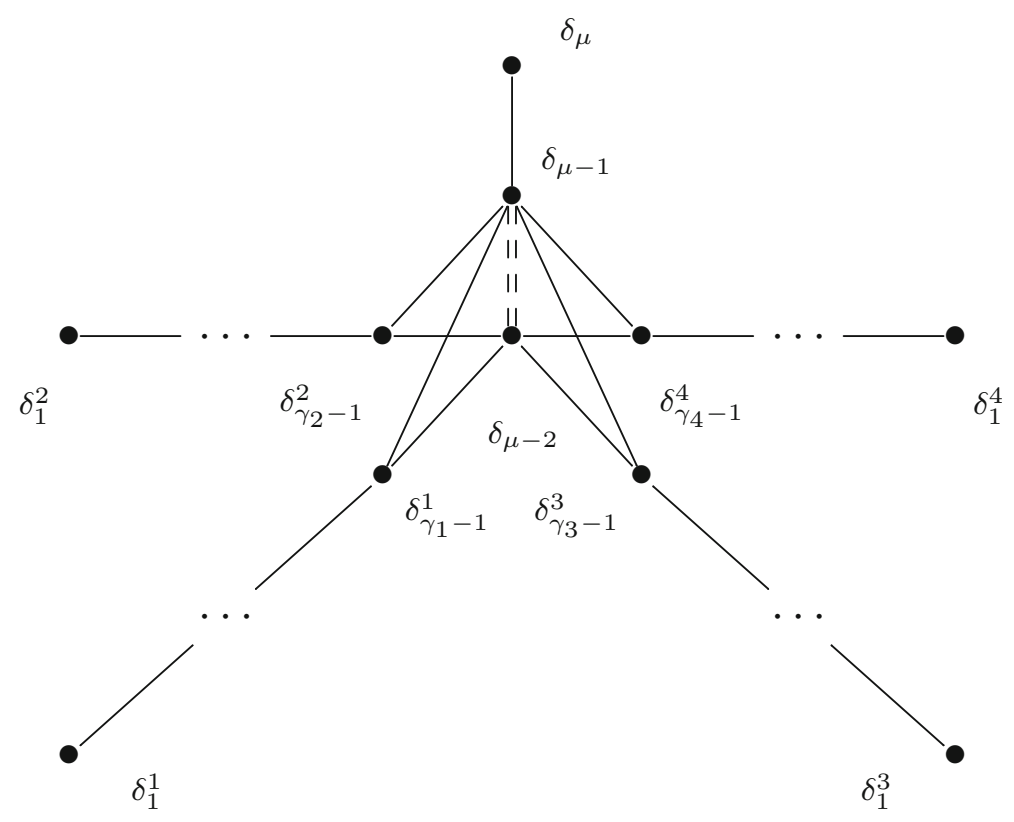

Fig. 2 The graph $S_{\gamma_{1}, \gamma_{2}, \gamma_{3}, \gamma_{4}}$

in the proof of [6, Proposition 3.6.2] which was corrected in [7].) By Proposition 7 we get

$$
\prod_{j=1}^{2} \bar{\zeta}_{X, j}(t)=\Delta\left(S_{\gamma_{1}, \gamma_{2}, \gamma_{3}, \gamma_{4}}\right)(t)
$$

This also gives an interpretation of the characteristic polynomial of the Coxeter element $c^{b}$ considered in [7].

A $k=0$ element of one of the series can again be given as the zero set of two weighted homogeneous functions of weights $w_{1}, w_{2}, w_{3}, w_{4}$ and degrees $d_{1}, d_{2}$. We are now ready to state the following analogue of [13, Theorem 6]:

Theorem 8 Let $X$ be a virtual ICIS and $\tilde{X}_{0}$ be the $k=0$ element of the dual series. Then we have

$$
\prod_{j=1}^{2} \bar{\zeta}_{X, j}(t)=P_{\widetilde{X}_{0}}(t) \cdot \operatorname{Or}_{\widetilde{X}_{0}}(t)=\prod_{j=1}^{2} \bar{\zeta}_{\widetilde{X}_{0}, j}^{*}(t)
$$

Proof By Equation (7.1), the left-hand side of Equation (7.2) is equal to $\Delta\left(S_{\gamma_{1}, \gamma_{2}, \gamma_{3}, \gamma_{4}}\right)(t)$. By [6, p. 98] (unfortunately, there is a misprint), there is the following formula for this polynomial : 


$$
\begin{aligned}
\Delta\left(S_{\gamma_{1}, \gamma_{2}, \gamma_{3}, \gamma_{4}}\right)(t)= & \left(t^{3}-2 t^{2}-2 t+1\right) \prod_{i=1}^{4} \frac{t^{\gamma_{i}}-1}{t-1} \\
& +t^{2} \sum_{i=1}^{4} \frac{t^{\gamma_{i}-1}-1}{t-1} \prod_{j=1, j \neq i}^{4} \frac{t^{\gamma_{j}}-1}{t-1}
\end{aligned}
$$

Using this formula, we can compute the polynomial $\prod_{j=1}^{2} \bar{\zeta}_{X, j}(t)$ in each case. The result is given in Table 3 . Under a certain non-degeneracy condition, the function $\zeta_{X, 2}(t)$ can also be computed by the formula of [16, Theorem 4] from the Newton polytope.

On the other hand, we can compute the Poincare series of $\widetilde{X}_{0}$ from the weights and degrees given in Table 1 . The polynomial $\operatorname{Or}_{\tilde{X}_{0}}(t)$ is given by

$$
\operatorname{Or}_{\tilde{X}_{0}}(t)=(1-t)^{-2} \prod_{i=1}^{4}\left(1-t^{\gamma_{i}}\right)
$$

where $\gamma_{1}, \gamma_{2} ; \gamma_{3}, \gamma_{4}$ are the Gabrielov numbers of $X$ which are the Dolgachev numbers of $\widetilde{X}$ (and of $\widetilde{X}_{0}$ ). The result is also given in Table 3 . Comparing these polynomials, we obtain the first equality of Equation (7.2).

The second equality follows from [9, Theorem].

Remark 9 The spectrum of an ICIS was defined in [11]. In a similar way one can define the spectrum of a virtual quadrangle complete intersection singularity $X$. Spectra for the series of ICIS above have been calculated by Steenbrink [20]. The spectrum of a virtual quadrangle complete intersection singularity agrees with the spectrum defined by setting $k=-1$ in the corresponding formulas of [20]. The numbers $e^{2 \pi \sqrt{-1} \alpha}$, where $\alpha$ is a number of this spectrum, coincide with the roots of $\prod_{j=1}^{2} \bar{\zeta}_{X, j}(t)$.

Acknowledgements This work has been partially supported by DFG. The second named author is also supported by JSPS KAKENHI Grant number JP16H06337. The authors would like to thank the referees for their useful comments.

Funding Open Access funding enabled and organized by Projekt DEAL.

Open Access This article is licensed under a Creative Commons Attribution 4.0 International License, which permits use, sharing, adaptation, distribution and reproduction in any medium or format, as long as you give appropriate credit to the original author(s) and the source, provide a link to the Creative Commons licence, and indicate if changes were made. The images or other third party material in this article are included in the article's Creative Commons licence, unless indicated otherwise in a credit line to the material. If material is not included in the article's Creative Commons licence and your intended use is not permitted by statutory regulation or exceeds the permitted use, you will need to obtain permission directly from the copyright holder. To view a copy of this licence, visit http://creativecommons.org/licenses/by/4.0/.

\section{References}

1. Arnold, V.I.: Normal forms of functions in neighbourhoods of degenerate critical points. Usp. Mat. Nauk 29, 11-49 (1974). Engl. translation in Russ. Math. Surv. 29, 19-48 (1974) 
2. Arnold, V.I.: Critical points of smooth functions and their normal forms. Usp. Mat. Nauk. 30(5), 3-65 (1975). Engl. translation in Russ. Math. Surv. 30:5, 1-75 (1975)

3. Berglund, P., Henningson, M.: Landau-Ginzburg orbifolds, mirror symmetry and the elliptic genus. Nucl. Phys. B 433, 311-332 (1995)

4. Berglund, P., Hübsch, T.: A generalized construction of mirror manifolds. Nucl. Phys. B 393, 377-391 (1993)

5. Decker, W., Greuel, G.-M., Pfister, G., Schönemann, H.: SINGULAR 4-1-0—a computer algebra system for polynomial computations. http://www.singular.uni-kl.de (2016)

6. Ebeling, W.: The Monodromy Groups of Isolated Singularities of Complete Intersections. Lect. Notes in Math., vol. 1293. Springer, Berlin (1987)

7. Ebeling, W.: Strange duality, mirror symmetry, and the Leech lattice. In: Bruce, J.W., Mond, D. (eds.) Singularity Theory, Proceedings of the European Singularities Conference, Liverpool 1996, London Math. Soc. Lecture Note Ser., vol. 263, pp. 55-77. Cambridge University Press, Cambridge (1999)

8. Ebeling, W.: The Poincaré series of some special quasihomogeneous surface singularities. Publ. Res. Inst. Math. Sci. 39(2), 393-413 (2003)

9. Ebeling, W., Gusein-Zade, S.M.: Monodromies and Poincaré series of quasihomogeneous complete intersections. Abh. Math. Sem. Univ. Hambg. 74, 175-179 (2004)

10. Ebeling, W., Gusein-Zade, S.M.: Saito duality between Burnside rings for invertible polynomials. Bull. Lond. Math. Soc. 44, 814-822 (2012)

11. Ebeling, W., Steenbrink, J.H.M.: Spectral pairs for isolated complete intersection singularities. J. Algebraic Geom. 7, 55-76 (1998)

12. Ebeling, W., Takahashi, A.: Strange duality of weighted homogeneous polynomials. Compositio Math. 147, 1413-1433 (2011)

13. Ebeling, W., Takahashi, A.: Strange duality between hypersurface and complete intersection singularities. Arnold Math. J. 2, 277-298 (2016)

14. Ebeling, W., Takahashi, A.: Graded matrix factorizations of size two and reduction. Preprint arXiv:2101.05075

15. Ebeling, W., Wall, C.T.C.: Kodaira singularities and an extension of Arnold's strange duality. Compositio Math. 56, 3-77 (1985)

16. Gusev, G.G.: The zeta-function of a polynomial on a complete intersection and Newton polytopes. Algebra i Analiz 23(3), 137-149 (2011). Engl. translation in St. Petersburg Math. J. 23, no. 3, 511-519 (2012)

17. Kouchnirenko, A.G.: Polyèdres de Newton et nombres de Milnor. Invent. Math. 32, 1-31 (1976)

18. Saito, K.: Duality for regular systems of weights: a précis. In: Kashiwara, M., Matsuo, A., Saito, K., Satake, I. (eds.) Topological Field Theory, Primitive Forms and Related Topics, Progress in Math., vol. 160, pp. 379-426. Birkhäuser, Boston (1998)

19. Saito, K.: Duality for regular systems of weights. Asian J. Math. 2(4), 983-1047 (1998)

20. Steenbrink, J. H. M.: Spectra of $\mathcal{K}$-unimodal isolated singularities of complete intersections. In: Bruce, J.W., Mond, D. (eds.) Singularity Theory, Proceedings of the European Singularities Conference, Liverpool 1996, London Math. Soc. Lecture Note Ser., vol. 263, pp. 151-162. Cambridge University Press, Cambridge (1999)

21. Wall, C.T.C.: Classification of unimodal isolated singularities of complete intersections. Proc. Symp. Pure Math. 40(Part 2), 625-640 (1983)

22. Wall, C.T.C.: Notes on the classification of singularities. Proc. Lond. Math. Soc. (3) 48(3), 461-513 (1984)

23. Wall, C.T.C.: Elliptic complete intersection singularities. In: Mond, D., Montaldi, J. (eds.) Singularity Theory and its Applications, Part I, Warwick 1989, Lecture Notes in Math., vol. 1462, pp. 340-372. Springer, Berlin (1991)

Publisher's Note Springer Nature remains neutral with regard to jurisdictional claims in published maps and institutional affiliations. 